Diamagnetic drift stabilized ballooning modes in a 3D heliotron

This article has been downloaded from IOPscience. Please scroll down to see the full text article.

2012 Plasma Phys. Control. Fusion 54014006

(http://iopscience.iop.org/0741-3335/54/1/014006)

View the table of contents for this issue, or go to the journal homepage for more

Download details:

IP Address: 203.129.48.64

The article was downloaded on 11/12/2011 at 11:19

Please note that terms and conditions apply. 


\title{
Diamagnetic drift stabilized ballooning modes in a 3D heliotron
}

\author{
D Barmaz ${ }^{1}$, W A Cooper ${ }^{1}$, B F McMillan ${ }^{1}$ and R L Dewar ${ }^{2}$ \\ ${ }^{1}$ Ecole Polytechnique Fédérale de Lausanne (EPFL), Centre de Recherches en Physique des Plasmas, \\ CH1015 Lausanne, Switzerland \\ ${ }^{2}$ Department of Theoretical Physics, Research School of Physical Sciences and Engineering, \\ The Australian University, Canberra 2600, Australia \\ E-mail: david.barmaz@gmail.com
}

Received 18 September 2010, in final form 31 January 2011

Published 8 December 2011

Online at stacks.iop.org/PPCF/54/014006

\begin{abstract}
An analysis of kinetic ballooning modes is presented, ray trajectories for the inward shifted Large Helical Device configuration at modest $\langle\beta\rangle$ and with an anisotropic pressure profile due to the hot particles are evaluated and an estimate of the marginal stability is computed using the Weyl formula. The Weyl formula indicates that the finite diamagnetic drifts of the hot particles displaces the minimum phase space volume to lower frequencies; however, its magnitude is higher than when these drift effects are ignored.
\end{abstract}

(Some figures may appear in colour only in the online journal)

\section{Introduction}

In the Large Helical Device (LHD) [1], high- $\langle\beta\rangle$ can be reached at low density operation. In this configuration, the pressure anisotropy driven by the neutral beam ions can be very significant with $p_{\|}>p_{\perp}$ [2]. This is expected because LHD has a powerful tangential neutral beam injection system. When the density increases, the electron temperature becomes lower and the slowing down time becomes shorter, which induces a decrease in the pressure anisotropy [2].

A first interesting observation that is worth mentioning is that, in the ideal MHD model, the ballooning instabilities (which are one of the most important sort of instabilities that appear in a stellarator, due to the interaction of pressure gradients with the magnetic field line curvature) in heliotron are expected to appear well below the $\langle\beta\rangle$ values achieved in LHD [3].

Another observation reported by Watanabe [4] is that the experimental data obtained with LHD are found to better agree with the theoretical predictions of ideal MHD when the hot particle $\langle\beta\rangle$ gradients are ignored. This tends to suggest that the modeling of the stability properties of LHD may be improved by extending the ideal MHD model.

Kinetic effects can be invoked to try to reconcile the experimental observations with the theoretical expectations. Diamagnetic-drift-frequency stabilizing effects on ballooning modes have already been investigated for tokamak geometry [5]. McMillan and Dewar [6] concentrated their efforts on stellarator geometry. They showed that finite Larmor radius effects are often sufficient to stabilize the plasma. They used semiclassical techniques to analyse these effects. In this study, their model is extended by considering the drift stabilization term due to the presence of anisotropic hot ions $\omega^{*}=\Omega_{\mathrm{pi}}^{*} k_{\alpha}+\Omega_{\mathrm{h}}^{*} / k_{\alpha}$ varying across the plasma volume and a frequency ordering in which the drift motion of the rapid fast particles causes them to be weakly interacting in the dynamics of the mode. A typical MHD equilibrium heated by tangential neutral beam injectors is considered. This enabled the computation of more realistic growth rates and frequencies to model ballooning stability.

A kinetic ballooning mode analysis that includes finite thermal ion diamagnetic drifts, compressibility and trapped electron effects predicts that lower $n$ instabilities in LHD are weakened but higher $n$ modes remain unstable [7]. Yamagishi et al have also included ballooning ray tracing in their analysis but do not treat an anisotropic fast ion species and do not evaluate the Weyl number [6].

We begin the analysis by reviewing the coordinate system (section 2) used and the theoretical background (section 3) leading to the ray equations (section 4). To this end, we resort to a drift-magnetohydrodynamic model developed by Cooper [8] in which the fast particles' drifts are much larger than the typical mode growth rates in the ballooning limit. Then we briefly explain our numerical approach (section 5) and study 
ray trajectories (section 6) in the kinetic ballooning model. We finally end up with an estimate for marginal stability using the Weyl formula (section 7).

\section{Coordinate system}

In this paper, we use the standard ballooning coordinate system described in [9]. The flux surface is parametrized by the poloidal angle $\theta$ and the field line label $\alpha$, which varies between 0 and $2 \pi$. The safety factor $q$ is taken as the radial coordinate.

\section{Kinetic ballooning modes}

Considering the linearized gyrokinetic equation [10] in ballooning space and following Brocher et al [11], we choose a configuration where the drift effects of energetic and thermal species play a role and where trapped particle effects are neglected. Using the approximation $\omega \ll \omega_{\mathrm{dh}}$ where $\omega_{\mathrm{dh}}$ is the drift frequency of hot particles, we can write, in the variational form [8],

$$
\begin{aligned}
& \int_{-\infty}^{\infty} \mathrm{d} \theta\left\{\frac{\sigma k_{\perp}^{2}}{\sqrt{g} B^{2}}|\sqrt{g} \boldsymbol{B} \cdot \nabla \chi|^{2}-\frac{k_{\alpha} \sqrt{g} p^{\prime}(s)}{\psi^{\prime}(s) B^{2}}\right. \\
&\left.\cdot\left[\left(1+\frac{\sigma}{\tau}\right) \boldsymbol{B} \times \boldsymbol{k}_{\perp} \cdot \boldsymbol{\kappa}-\left.\frac{k_{\alpha}}{\psi^{\prime}(s)} \frac{\partial p_{\perp \mathrm{h}}}{\partial s}\right|_{B}\right]|\chi|^{2}\right\} \\
&=\int_{-\infty}^{\infty} \mathrm{d} \theta M_{\mathrm{i}} N_{\mathrm{i}} \frac{\sqrt{g} k_{\perp}^{2}}{B^{2}} \omega\left[\omega-\left(\omega_{\mathrm{pi}}^{*}+\frac{M_{\mathrm{h}} N_{\mathrm{h}} \omega_{\mathrm{h}}^{*}}{M_{\mathrm{i}} N_{\mathrm{i}} b_{\mathrm{h}}}\right)\right]|\chi|^{2},
\end{aligned}
$$

where $\boldsymbol{B}$ is the magnetic field, $\boldsymbol{\kappa}$ the magnetic field line curvature, $p$ the thermal pressure, $p_{\perp \mathrm{h}}$ the hot particle perpendicular pressure, ' denotes the derivative with respect to $s, M_{\mathrm{i}}$ and $M_{\mathrm{h}}$ the thermal ion, respectively, hot ion mass, $N_{\mathrm{i}}$ and $N_{\mathrm{h}}$ the thermal ion and the hot ion density and $b_{\mathrm{h}}$ the Larmor radius parameter $[10,11]$ defined as

$$
b_{\mathrm{h}}=\frac{k_{\perp}^{2}}{B^{2}} \frac{p_{\perp \mathrm{h}} M_{\mathrm{h}}}{Z_{\mathrm{h}}^{2} e^{2} N_{\mathrm{h}}},
$$

where $Z_{\mathrm{h}}$ is the hot ion atomic number and $e$ the elementary charge. $\omega_{\mathrm{pi}}^{*}$ is the thermal ion hot diamagnetic frequency and $\omega_{\mathrm{h}}^{*}$ the hot ion diamagnetic drift frequency. $0 \leqslant s \leqslant 1$ denotes the radial coordinate proportional to the normalized toroidal magnetic flux. In the ballooning ordering, the wave vector $\boldsymbol{k}$ (commonly written $k=k_{\alpha} \nabla \alpha+k_{q} \nabla q$ ) is taken to be large. The frequency $\omega$ is taken to be finite and the product $\boldsymbol{k} \cdot \boldsymbol{B} \approx 0$. We then solve the previous equation in the single-fluid ballooning limit $\left(\omega_{\mathrm{pi}}^{*}=0\right.$ and $\left.\omega_{\mathrm{h}}^{*}=0\right)$, and obtain a dispersion relation for this model:

$$
\omega^{2}=\lambda\left(\alpha, q, \theta_{k}\right),
$$

where $\theta_{k}=k_{q} / k_{\alpha}$ corresponds to the radial wavenumber. This $\lambda\left(\alpha, q, \theta_{k}\right)$ has the following periodic properties [9]:

$$
\lambda\left(\alpha+2 \pi, q, \theta_{k}\right)=\lambda\left(\alpha, q, \theta_{k}\right)
$$

and

$$
\lambda\left(\alpha-2 \pi q, q, \theta_{k}+2 \pi\right)=\lambda\left(\alpha, q, \theta_{k}\right) .
$$

For simplicity reasons, we perform a change of variable: $\phi_{k}=\alpha+q \theta_{k}$. The periodicity relations then become

$$
\lambda\left(\phi_{k}+2 \pi, q, \theta_{k}\right)=\lambda\left(\phi_{k}, q, \theta_{k}\right)
$$

and

$$
\lambda\left(\phi_{k}, q, \theta_{k}+2 \pi\right)=\lambda\left(\phi_{k}, q, \theta_{k}\right) .
$$

It is shown [6] that the ballooning ordering can also account for effects beyond ideal MHD. For example, it can be applied to general plasma models such as the Maxwell-Vlasov equations. In this particular case, two specific effects are present, one corresponding to the trapped particle effects and the other one related to the finite gyro-radius term. The kinetic ballooning model is obtained by replacing

$$
\omega^{2} \rightarrow \omega\left(\omega+\omega_{\mathrm{pi}}^{*}+\omega_{\mathrm{h}_{\mathrm{eff}}}^{*}\right)
$$

in the ideal MHD ballooning equations. $\omega_{\mathrm{pi}}^{*}$ is the thermal ion diamagnetic drift frequency defined by [11]

$$
\omega_{\mathrm{pi}}^{*}=-k_{\alpha} \frac{p^{\prime}(s)}{2 Z_{\mathrm{i}} e \psi^{\prime}(s) N_{\mathrm{i}}} \approx k_{\alpha} \Omega_{\mathrm{pi}}^{*}
$$

and $\omega_{\mathrm{h}_{\text {eff }}}^{*}$ is the hot ion diamagnetic drift frequency [11]

$$
\begin{aligned}
\omega_{\mathrm{h}_{\mathrm{eff}}}^{*} & =\left\langle\frac{M_{\mathrm{h}} \omega_{\mathrm{h}}^{*}}{M_{\mathrm{i}} N_{\mathrm{i}} b_{\mathrm{h}}}\right\rangle \\
= & \frac{\int_{-\infty}^{\infty} \mathrm{d} \theta \frac{\sqrt{g} k_{\perp}^{2} M_{\mathrm{h}} N_{\mathrm{h}} \omega_{\mathrm{h}}^{*}}{\psi^{\prime}(s) B^{2} M_{\mathrm{i}} N_{\mathrm{i}} b_{\mathrm{h}}}|\chi|^{2}}{\int_{-\infty}^{\infty} \mathrm{d} \theta \frac{\sqrt{g} k_{\perp}^{2}}{\psi^{\prime}(s) b^{2}}|\chi|^{2}} \Omega_{\mathrm{h}}^{*},
\end{aligned}
$$

where $\omega_{\mathrm{h}}^{*}$ is given by

$$
\omega_{\mathrm{h}}^{*}=-\left.k_{\alpha} \frac{p_{\perp \mathrm{h}}}{Z_{\mathrm{h}} e \psi^{\prime}(s) N_{\mathrm{h}}^{2}} \frac{\partial N_{\mathrm{h}}}{\partial s}\right|_{B}
$$

with $p(s)$ the thermal pressure, $N_{\mathrm{h}}$ the hot particle density and $N_{\mathrm{i}}$ the thermal ion density. To obtain the second relation, $k_{\alpha}$ is taken sufficiently large consistent with ballooning theory. We can thus neglect the $1 / k_{\alpha}^{2}$ term in the $\omega_{\mathrm{h}_{\mathrm{eff}}}^{*}$ expansion. The kinetic effect is equivalent to a simple frequency shift on each field line and the eigensolution of the ballooning equation is conserved. Thus, we can calculate the local kinetic growth rates using the substitution presented in equation (8) in the local ideal MHD dispersion relation. Following McMillan [6], we write the dispersion relation for the drift stabilized MHD ballooning model

$$
\Lambda\left(k_{\alpha}, k_{q}, \alpha, q, \omega\right)=\omega_{\mathrm{MHD}}^{2}-\omega\left(\omega-k_{\alpha} \Omega_{\mathrm{pi}}^{*}-\frac{1}{k_{\alpha}} \Omega_{\mathrm{h}}^{*}\right)=0 .
$$

The frequency $\omega$ can take two different values:

$$
\omega=\frac{k_{\alpha} \Omega_{\mathrm{pi}}^{*}+\frac{1}{k_{\alpha}} \Omega_{\mathrm{h}}^{*}}{2}\left[1+\left(1+\frac{4 \omega_{\mathrm{MHD}}^{2}}{\left(k_{\alpha} \Omega_{\mathrm{pi}}^{*}+\frac{1}{k_{\alpha}} \Omega_{\mathrm{h}}^{*}\right)^{2}}\right],\right.
$$


which corresponds to the drift branch and

$\omega=\frac{k_{\alpha} \Omega_{\mathrm{pi}}^{*}+\frac{1}{k_{\alpha}} \Omega_{\mathrm{h}}^{*}}{2}\left[1-\left(1+\frac{4 \omega_{\mathrm{MHD}}^{2}}{\left(k_{\alpha} \Omega_{\mathrm{pi}}^{*}+\frac{1}{k_{\alpha}} \Omega_{\mathrm{h}}^{*}\right)^{2}}\right)^{1 / 2}\right]$,

which corresponds to the MHD branch.

\section{Ray equations}

We extended the ray equation obtained in [6] to the weakly interacting hot particle model we are considering. Thus, the drift frequency $\Omega^{*}$ is not constant anymore, but varies in the $\left(q, \alpha, \theta_{k}\right)$ space. We derive the four ray equations from the dispersion relation (12):

$$
\begin{aligned}
\dot{\alpha} & =\frac{\partial \Lambda}{\partial k_{\alpha}} \\
& =\omega \Omega_{\mathrm{pi}}^{*}-\frac{\theta_{k}}{k_{\alpha}} \frac{\partial\left(\omega_{\mathrm{MHD}}^{2}\right)}{\partial \theta_{k}}-\frac{\omega}{k_{\alpha}^{2}} \Omega_{\mathrm{h}}^{*}-\frac{\omega}{k_{\alpha}^{2}} \frac{\partial \Omega_{\mathrm{h}}^{*}}{\partial \theta_{k}}-\omega \frac{\partial \Omega_{\mathrm{pi}}^{*}}{\partial \theta_{k}},
\end{aligned}
$$

$\dot{q}=\frac{\partial \Lambda}{\partial k_{q}}=\frac{1}{k_{\alpha}} \frac{\partial\left(\omega_{\mathrm{MHD}}^{2}\right)}{\partial \theta_{k}}+\omega \frac{\partial \Omega_{\mathrm{pi}}^{*}}{\partial \theta_{k}}+\frac{\omega}{k_{\alpha}^{2}} \frac{\partial \Omega_{\mathrm{h}}^{*}}{\partial \theta_{k}}$,

$\dot{k_{\alpha}}=-\frac{\partial \Lambda}{\partial \alpha}=-\frac{\partial\left(\omega_{\mathrm{MHD}}^{2}\right)}{\partial \alpha}-\frac{\omega}{k_{\alpha}} \frac{\partial \Omega_{\mathrm{h}}^{*}}{\partial \alpha}-\omega k_{\alpha} \frac{\partial \Omega_{\mathrm{pi}}^{*}}{\partial \alpha}$,

$\dot{k_{q}}=-\frac{\partial \Lambda}{\partial q}=-\frac{\partial\left(\omega_{\mathrm{MHD}}^{2}\right)}{\partial q}-\omega k_{\alpha} \frac{\partial \Omega_{\mathrm{pi}}^{*}}{\partial q}-\frac{\omega}{k_{\alpha}} \frac{\partial \Omega_{\mathrm{h}}^{*}}{\partial q}$.

This shows that taking into account the variation of the drift frequency strongly modifies the ray equations.

\section{Numerical implementation}

To study the ballooning instabilities, we used data extracted from the BECOOL [12] program. The original BECOOL program was slightly modified to get $\omega_{\mathrm{MHD}}^{2}, \Omega_{\mathrm{pi}}^{*}$ and $\Omega_{\mathrm{h}}^{*}$ as a function of the three coordinates $\phi_{k}, q$ and $\theta_{k}$. We used the data extracted from BECOOL to obtain a Fourier series in $\theta_{k}$ and $\phi_{k}$, and used a spline interpolation for the $q$ coordinate. We computed the derivatives in each point of the phase space and followed ray trajectories using a Runge-Kutta integration scheme, applying a small correction to ensure that $\omega$ stays constant.

\section{Ray trajectory}

\subsection{Initial data}

As input values for BECOOL, we used data corresponding to the inward shifted Large Helical Device (LHD) configuration at modest $\langle\beta\rangle$ [11]. The fast particle species are described using an anisotropic pressure bi-Maxwellian distribution function model. The $\left\langle\beta_{\text {dia }}\right\rangle$ value is given by $\left\langle\beta_{\text {dia }}\right\rangle \approx 1.6 \%$. The following parameters were used:

- Thermal pressure: $p(s)=p(0)(1-s)\left(1-s^{4}\right)$

- Hot particle pressure amplitude: $p_{\mathrm{h}}(s)=p_{\mathcal{H}}(1-s)$

- Anisotropy factor: $\left[\frac{T_{\perp}}{T_{\|}}\right](s)=\left[\frac{T_{\perp}}{T_{\|}}\right](0)\left(1-s^{2}\right)$

- Toroidal current $J(s)=0$.

Input values corresponding to these parameters were used in BECOOL to compute $\omega_{\mathrm{MHD}}^{2}, \Omega_{\mathrm{pi}}^{*}$ and $\Omega_{\mathrm{h}}^{*}$. $\omega_{\mathrm{MHD}}^{2}$ reaches a minimum when $q \approx 1.36$ and for $\theta_{k} \approx \pi$. $\omega_{\mathrm{MHD}}^{2}$ mainly depends on $q$ and $\theta_{k}$. However, it also has a small dependence in $\phi_{k}$ (thus corresponding implicitly to the field line variable $\alpha)$. $\Omega_{\mathrm{pi}}^{*}$ does not depend directly on $\theta_{k}$ and $\phi_{k}$, but its value increases as $q$ increases. Finally, the $\Omega_{\mathrm{h}}^{*}$ value has a maximum around $q \approx 1.4$ and $\theta_{k}=\pi$ and is minimal for $\theta_{k}=0$. The behavior of $\Omega_{\mathrm{h}}^{*}$ is more or less opposite to the behavior of $\omega_{\mathrm{MHD}}^{2}$. The hot ion diamagnetic drift frequcency does not have a very strong dependence in $\phi_{k}$.

\subsection{Orbits in the $\left(\alpha, q, \theta_{k}\right)$ space}

Solving the ray equations and using the method explained in the previous paragraphs, we can now follow a ray trajectory. Note that the ray trajectories presented below correspond to the MHD branch only (see equation (14)). We only focus on this branch because the kinetic modifications that we included to the linearized gyrokinetic equation in ballooning space [10] are limited to diamagnetic drift extensions to the fluid MHD model. Figure 1 shows a projection in the $\left(q, \theta_{k}\right)$ plane of the ray trajectories for all $\alpha$ values, using different starting points for the simulation. We see that there are two types of orbits:

- closed orbits in the $\left(q, \theta_{k}\right)$ plane which correspond to cylindrical orbits in the $\left(\alpha, q, \theta_{k}\right)$ space. The cylinder is infinitely extended in the $\alpha$ direction.

- open orbits in the $\left(q, \theta_{k}\right)$ plane, which correspond to a plane in the $\left(\alpha, q, \theta_{k}\right)$ space.

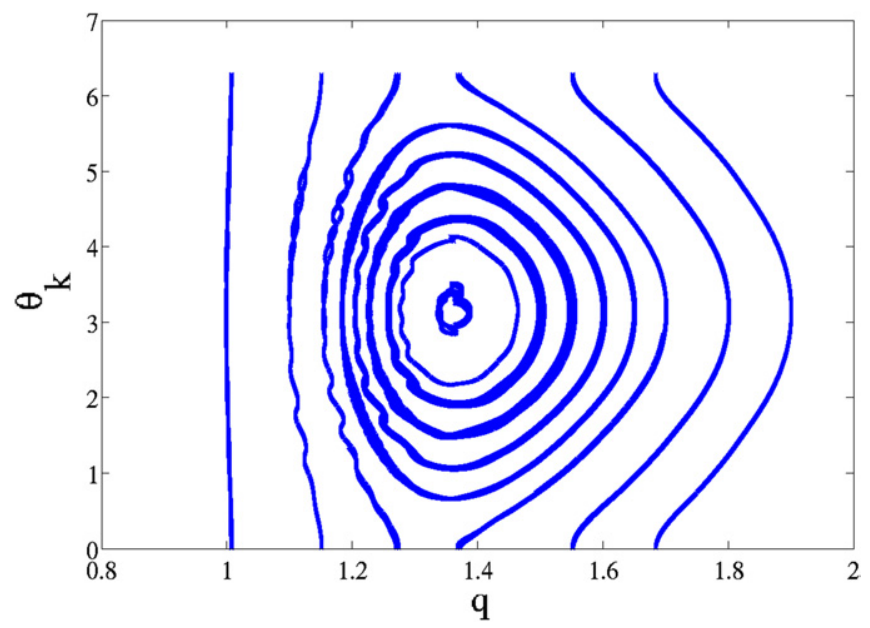

Figure 1. Projection of different ray trajectories superimposed in the $\left(q, \theta_{k}\right)$ plane for all $\alpha$ values. We notice two different types of orbits, closed orbits in the central region of the $\left(q, \theta_{k}\right)$ plane, and open orbits on the sides. 


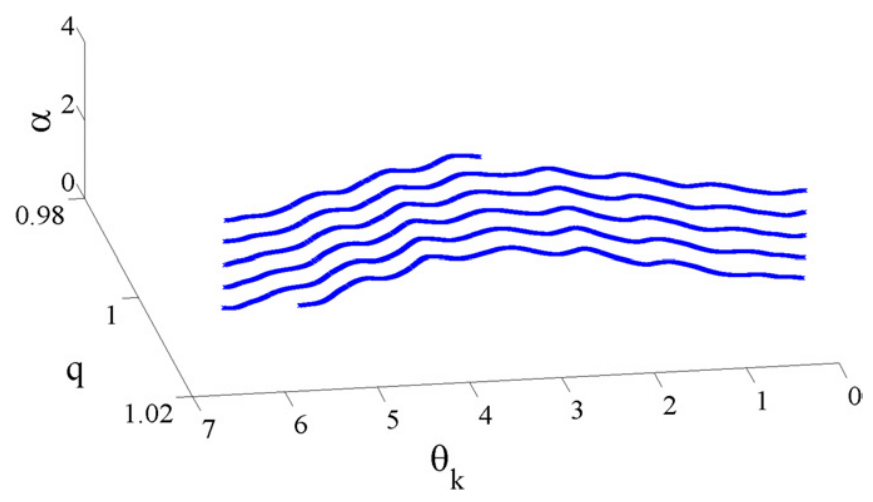

Figure 2. Typical ray trajectory for an orbit located in a plane in the $\left(\theta_{k}, q, \alpha\right)$ space.

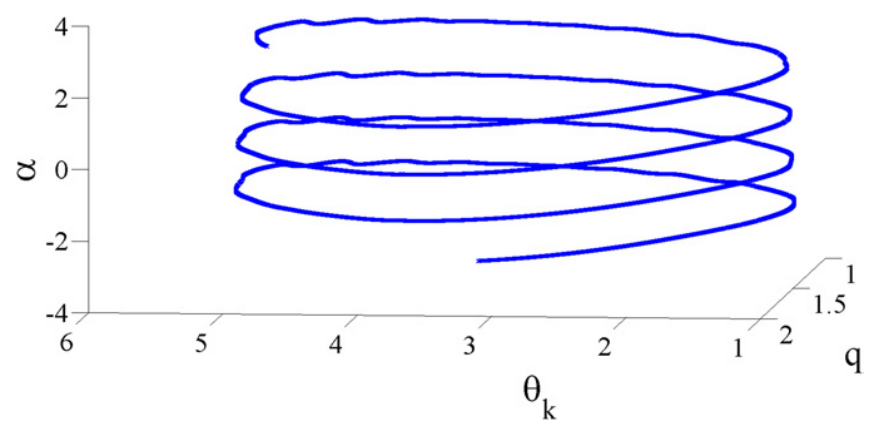

Figure 3. Typical ray trajectory for a spiral orbit in the $\left(\theta_{k}, q, \alpha\right)$ space. This kind of orbit, located on a cylinder in the $\left(\theta_{k}, q, \alpha\right)$ space, corresponds to initial values close to the minimum of $\omega_{\mathrm{MHD}}^{2}$.

Closed orbits correspond to initial values close to the minimum of $\omega_{\mathrm{MHD}}^{2}$ (i.e. close to $q \approx 1.36$ and $\theta_{k} \approx \pi$ ). The two different kinds of ray trajectories are shown in figures 2 and 3.

\subsection{Dominating terms in the ray equations}

A simple study of the derivatives as a function of time shows which terms are the dominant ones in the ray equations. For the MHD branch, the two most important terms in the $\dot{\alpha}$ equation are $-\left(\theta_{k} / k_{\alpha}\right) \partial \omega_{\mathrm{MHD}}^{2} / \partial \theta_{k}$ and $\omega \Omega_{\mathrm{pi}}^{*}$ (see figure 4$)$. The other ones can more or less be neglected. The two most important terms in the $\dot{q}$ equation are $\left(1 / k_{\alpha}\right) \partial\left(\omega_{\text {MHD }}^{2}\right) / \partial \theta_{k}$ and $\omega \cdot \partial \Omega_{\mathrm{pi}}^{*} / \partial \theta_{k}$. These two terms are always opposed to one another in the sense that they have almost the same magnitude but opposite signs. This can be seen in figure 5. In the $\dot{k_{\alpha}}$ equation, the two dominating terms are $-\partial\left(\omega_{\mathrm{MHD}}^{2}\right) / \partial \alpha$ and $-\omega k_{\alpha} \cdot \partial \Omega_{\mathrm{pi}}^{*} / \partial \alpha$ (figure 6). We see that these two terms more or less always cancel each other out. Finally for the $\dot{k_{q}}$ equation, the dominating term is $-\partial\left(\omega_{\mathrm{MHD}}^{2}\right) / \partial q$ (figure 7$)$. The two other terms are much smaller.

\section{Weyl formula}

The Weyl formula

$$
N_{\text {Weyl }}(w)=\frac{1}{(2 \pi)^{n}} \int \mathrm{d} x^{n} \mathrm{~d} k^{n}
$$

is used to estimate the number of quantized modes below some frequency $\omega$. It is based on the volume of phase space enclosed

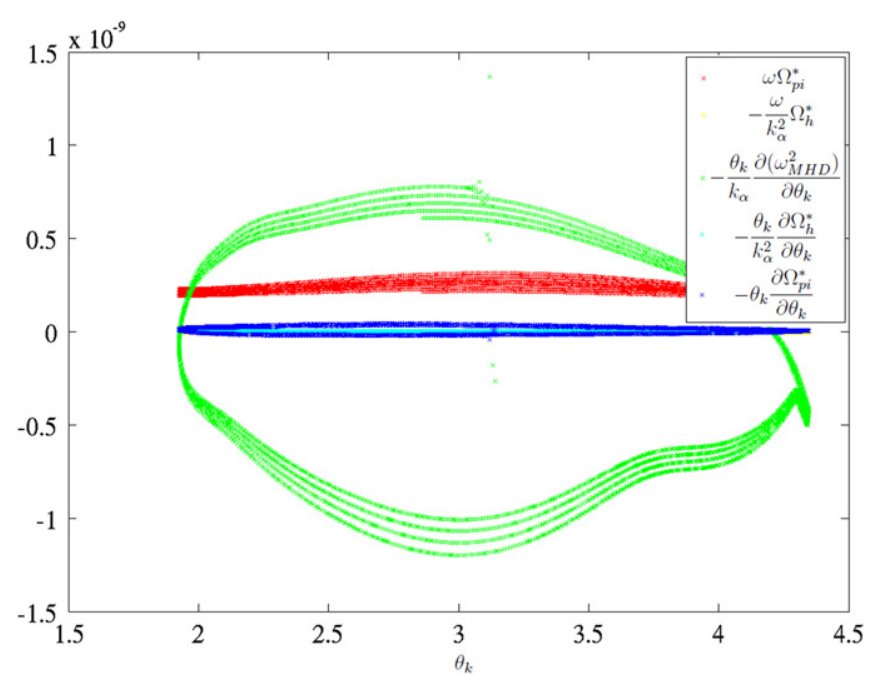

Figure 4. Importance of the terms that drive $\dot{\alpha}$ in the case of a close orbit in the $\left(q, \theta_{k}\right)$ plane.

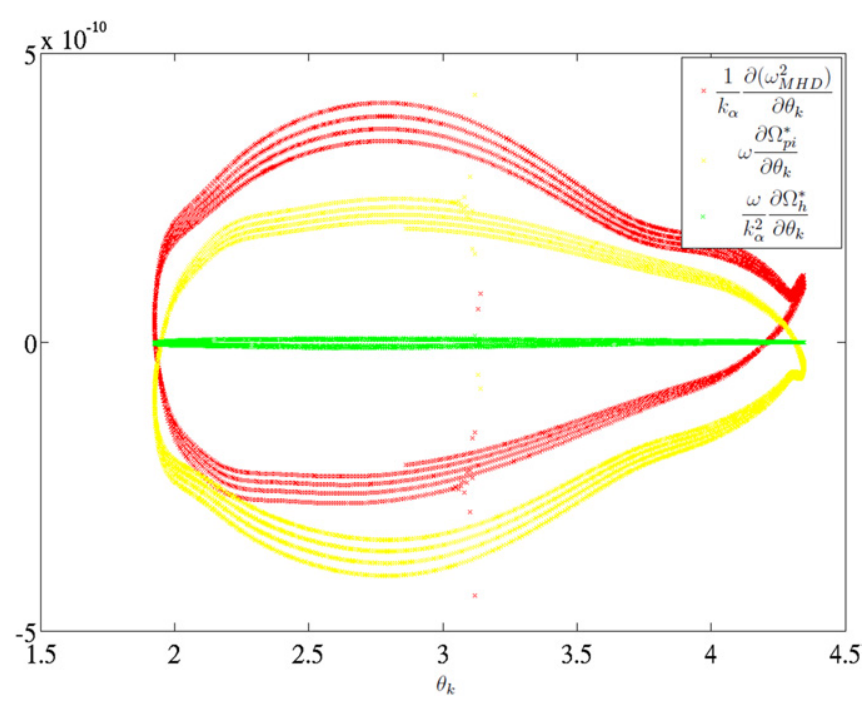

Figure 5. Importance of the terms that drive $\dot{q}$ in the case of a close orbit in the $\left(q, \theta_{k}\right)$ plane.

by the $\omega$ contour. As the standard derivation of the Weyl formula requires the dispersion relation to be real, we followed McMillan [6] and took

$$
E=\Omega_{\mathrm{pi}}^{*}\left(\omega, \theta_{k}, k_{\alpha}, \alpha, q, \Omega_{\mathrm{h}}^{*}\right) .
$$

The Weyl estimate for the number of modes in the spectrum of $\Omega_{\mathrm{pi}}^{*}$ above $\Omega_{\mathrm{pi}}^{*}$ is is then given by the integral of the Heaviside step function

$$
N_{\text {Weyl }}=\frac{1}{4 \pi^{2}} \int H\left(k_{\alpha}^{\max }-k_{\alpha}\right) \mathrm{d} V
$$

where

$k_{\alpha}^{\max }=\max \left\{\frac{\omega^{2}-\omega_{\mathrm{MHD}}^{2}}{2 \omega \Omega_{\mathrm{pi}}^{*}}\left[1 \pm\left(1-\frac{4 \omega^{2} \Omega_{\mathrm{pi}}^{*} \Omega_{\mathrm{h}}^{*}}{\left(\omega^{2}-\omega_{\mathrm{MHD}}^{2}\right)^{2}}\right)^{1 / 2}\right]\right\}$. 


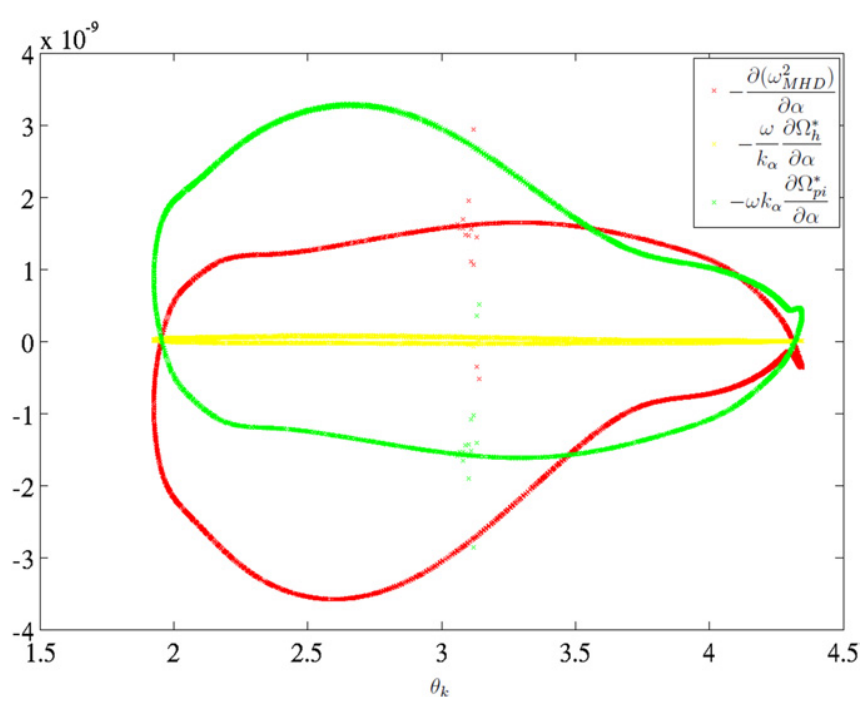

Figure 6. Importance of the terms that drive $\dot{k_{\alpha}}$ in the case of a close orbit in the $\left(q, \theta_{k}\right)$ plane.

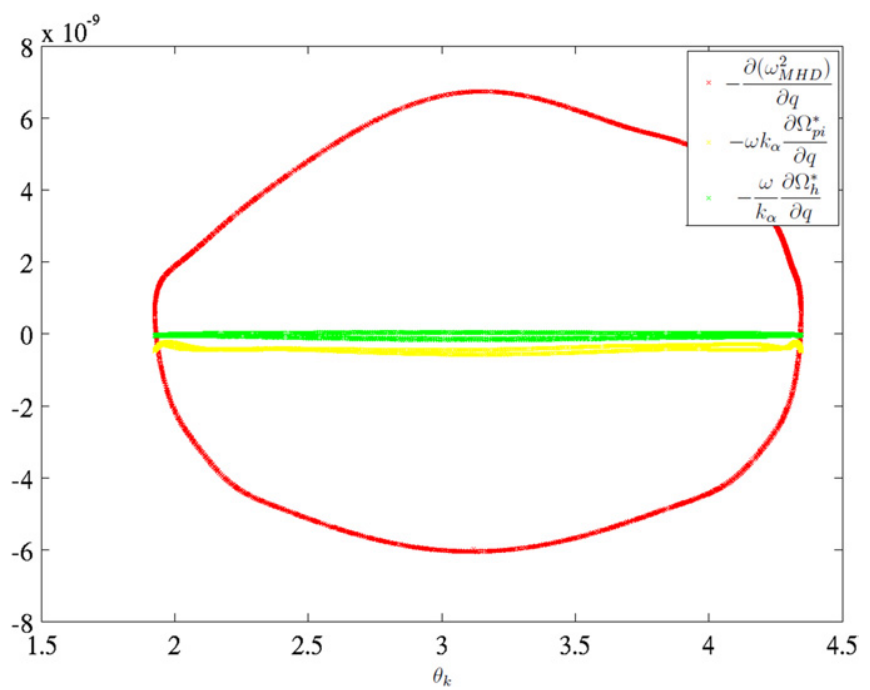

Figure 7. Importance of the terms that drive $\dot{k_{q}}$ in the case of a close orbit in the $\left(q, \theta_{k}\right)$ plane.

We suppose the term $\omega^{2} \Omega_{\mathrm{pi}}^{*} \Omega_{\mathrm{h}}^{*} /\left(\omega^{2}-\omega_{\mathrm{MHD}}^{2}\right)^{2}$ to be small. This happens in two cases:

- $\omega^{2} \gg \Omega_{\mathrm{pi}}^{*} \Omega_{\mathrm{h}}^{*} \gg\left|\omega_{\mathrm{MHD}}^{2}\right|$

- $\omega^{2} \ll\left|\omega_{\mathrm{MHD}_{\min }}^{2}\right| \ll \Omega_{\mathrm{pi}}^{*} \Omega_{\mathrm{h}}^{*}$.

We recall the expression previously obtained (section 3) for $\omega$ :

$\omega=\frac{1}{2}\left(k_{\alpha} \Omega_{\mathrm{pi}}^{*}+\frac{\Omega_{\mathrm{h}}^{*}}{k_{\alpha}}\right)\left[1 \pm\left(1+\frac{4 \omega_{\mathrm{MHD}}^{2}}{\left(k_{\alpha} \Omega_{\mathrm{pi}}^{*}+\frac{\Omega_{\mathrm{h}}^{*}}{k_{\alpha}}\right)^{2}}\right)^{1 / 2}\right]$ which can be expanded because $4 \omega_{\mathrm{MHD}}^{2} /\left(k_{\alpha} \Omega_{\mathrm{pi}}^{*}+\Omega_{\mathrm{h}}^{*} / k_{\alpha}\right)^{2}$ is small:

$$
\omega=\frac{1}{2}\left(k_{\alpha} \Omega_{\mathrm{pi}}^{*}+\frac{\Omega_{\mathrm{h}}^{*}}{k_{\alpha}}\right)\left[1 \pm\left(1+\frac{2 \omega_{\mathrm{MHD}}^{2}}{\left(k_{\alpha} \Omega_{\mathrm{pi}}^{*}+\frac{\Omega_{\mathrm{h}}^{*}}{k_{\alpha}}\right)^{2}}\right)\right] .
$$

This leads to two different values for $\omega$, depending on the sign:

$$
\begin{gathered}
\omega=k_{\alpha} \Omega_{\mathrm{pi}}^{*}+\frac{\Omega_{\mathrm{h}}^{*}}{k_{\alpha}}+\frac{\omega_{\mathrm{MHD}}^{2}}{k_{\alpha} \Omega_{\mathrm{pi}}^{*}+\frac{\Omega_{\mathrm{h}}^{*}}{k_{\alpha}}}, \\
\omega=-\frac{\omega_{\mathrm{MHD}}^{2}}{k_{\alpha} \Omega_{\mathrm{pi}}^{*}+\frac{\Omega_{\mathrm{h}}^{*}}{k_{\alpha}}} .
\end{gathered}
$$

The first value of $\omega$ corresponds to the drift branch, the second one corresponds to the MHD branch. If we come back to our $k_{\alpha}$ expansion, we see that $\omega^{2} \gg \Omega_{\mathrm{pi}}^{*} \Omega_{\mathrm{h}}^{*} \gg\left|\omega_{\mathrm{MHD}}^{2}\right|$ corresponds to equation (25) for $\omega$, while $\omega^{2} \ll\left|\omega_{\mathrm{MHD}_{\min }}\right| \ll \Omega_{\mathrm{pi}}^{*} \Omega_{\mathrm{h}}^{*}$ only happens if $\omega$ corresponds to equation (26). We can now expand $k_{\alpha}$

$$
k_{\alpha}^{\max }=\max \left\{\frac{\omega^{2}-\omega_{\mathrm{MHD}}^{2}}{2 \omega \Omega_{\mathrm{pi}}^{*}}\left[1 \pm\left(1-\frac{2 \omega^{2} \Omega_{\mathrm{pi}}^{*} \Omega_{\mathrm{h}}^{*}}{\left(\omega^{2}-\omega_{\mathrm{MHD}}^{2}\right)^{2}}\right)\right]\right\},
$$

$k_{\alpha}^{\max }=\frac{\omega^{2}-\omega_{\mathrm{MHD}}^{2}}{\omega \Omega_{\mathrm{pi}}^{*}}-\frac{\omega \Omega_{\mathrm{h}}^{*}}{\omega^{2}-\omega_{\mathrm{MHD}}^{2}}$

Coming back to equation (21), we have

$$
N_{\text {Weyl }}=\frac{1}{4 \pi^{2}} \int_{S} \frac{\left(k_{\alpha}^{\max }\right)^{2}}{2} \mathrm{~d} \theta_{k} \mathrm{~d} \alpha \mathrm{d} q
$$

and replacing $k_{\alpha}$

$N_{\mathrm{Weyl}}=\frac{1}{4 \pi^{2}} \int_{S} \frac{1}{2}\left(\frac{\omega^{2}-\omega_{\mathrm{MHD}}^{2}}{\omega \Omega_{\mathrm{pi}}^{*}}-\frac{\omega \Omega_{\mathrm{h}}^{*}}{\omega^{2}-\omega_{\mathrm{MHD}}^{2}}\right)^{2} \mathrm{~d} \theta_{k} \mathrm{~d} \alpha \mathrm{d} q$,

$N_{\text {Weyl }}=\frac{1}{4 \pi^{2}} V(\omega)$,

where the volume $S$ is the domain in the $\left(q, \alpha, \theta_{k}\right)$ space for which

$$
\left(\omega^{2}-\frac{\omega_{\mathrm{MHD}}^{2}}{\omega \Omega_{\mathrm{pi}}^{*}}-\frac{\omega \Omega_{\mathrm{h}}^{*}}{\omega^{2}-\omega_{\mathrm{MHD}}^{2}}\right)>0 .
$$

The integral was approximated by summing over $92 \times 64 \times 64$ intervals in the $\left(q, \alpha, \theta_{k}\right)$ space. The value of the volume $V$ as a function of $\omega$ is plotted in figures 8 and 9 (corresponding to the two different interesting $\omega$ ranges). We see that it reaches a minimum for $\omega=1.632 \times 10^{-8}$. This corresponds to the marginal stability condition. A convergence study (figure 10) shows that the minimum phase space volume as a function of $\omega$ strongly depends on the number of intervals and on the discrete points chosen to estimate the integral. More precise results would be obtained by increasing the number of intervals. 


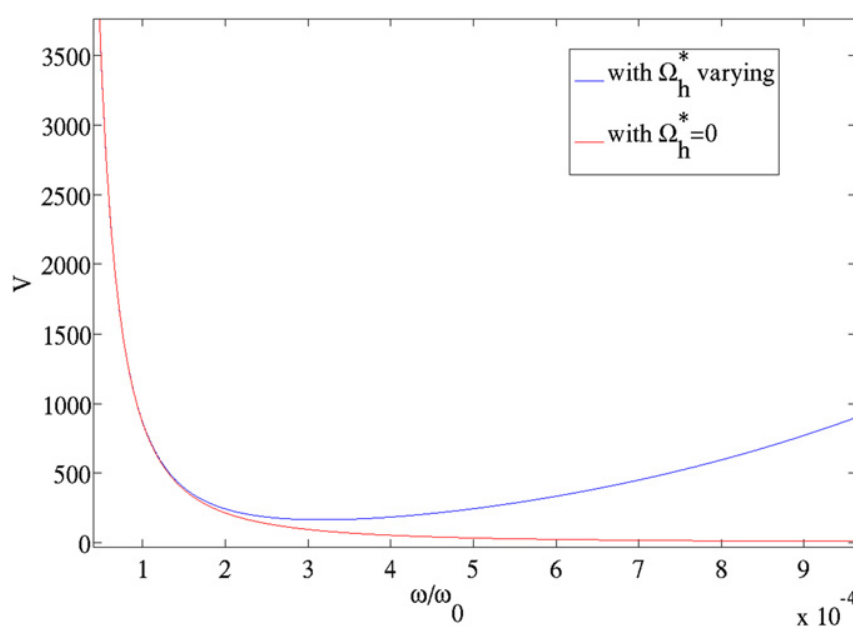

Figure 8. Value of the volume $V(\omega)$ for $\omega^{2} \ll\left|\omega_{\mathrm{MHD}_{\min }}^{2}\right|$ obtained by approximating the integral by a sum over $92 \times 64 \times 64$ intervals in the $\left(q, \alpha, \theta_{k}\right)$ space. The volume $V(\omega)$ is also computed in the case where the hot ion diamagnetic drift is neglected. Here $\omega_{0}=\sqrt{-\omega_{\mathrm{MHD}_{\min }^{2}}}$.

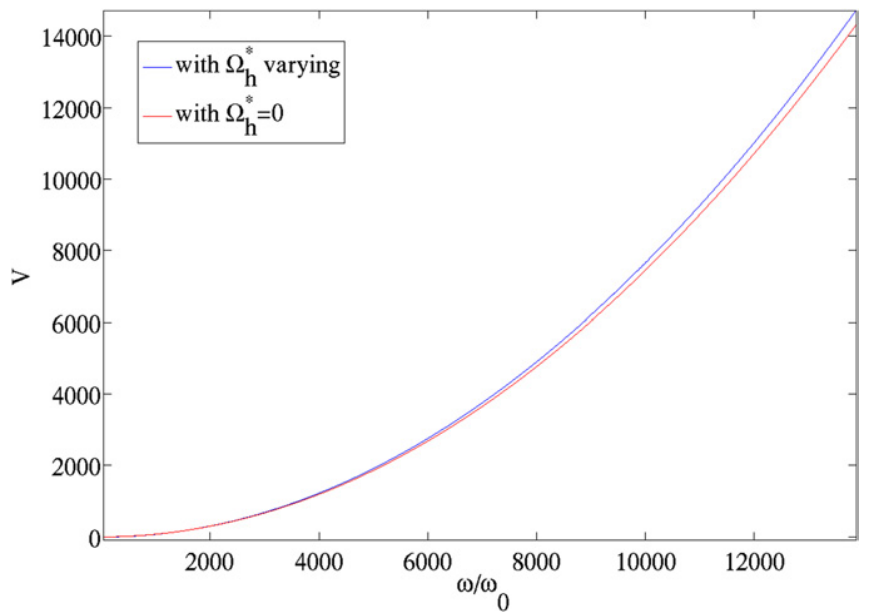

Figure 9. Value of the volume $V(\omega)$ for $\omega^{2} \gg \Omega_{\mathrm{pi}}^{*} \Omega_{\mathrm{h}}^{*}$ obtained by approximating the integral by a sum over $92 \times 64 \times 64$ intervals in the $\left(q, \alpha, \theta_{k}\right)$ space. The volume $V(\omega)$ is also computed in the case where the hot ion diamagnetic drift is neglected. Here $\omega_{0}=\sqrt{-\omega_{\mathrm{MHD}_{\min }}^{2}}$.

In figure 9, we see that the volume increases strongly for $\omega^{2} \gg \Omega_{\mathrm{pi}}^{*} \Omega_{\mathrm{h}}^{*}$. If we compare the results obtained with and without taking into account the hot particles drift, we see that the marginal stability corresponds to a $\omega$ value much bigger when the hot particles drift is neglected (figure 11): $\omega \approx 0.6 \sqrt{-\omega_{\mathrm{MHD}_{\min }}^{2}}$. The term $\omega \Omega_{\mathrm{h}}^{*} /\left(\omega^{2}-\omega_{\mathrm{MHD}}^{2}\right)$ in the integral is not very important for $\omega^{2} \gg \Omega_{\mathrm{pi}}^{*} \Omega_{\mathrm{h}}^{*}$ and for $\omega \ll \omega_{\mathrm{MHD}}^{2}$, but it becomes very big as $\omega^{2} \rightarrow \omega_{\mathrm{MHD}}^{2}$.

\section{Conclusion}

Strongly localized ballooning modes are often the first instabilities to become noticeable in a fusion device such as

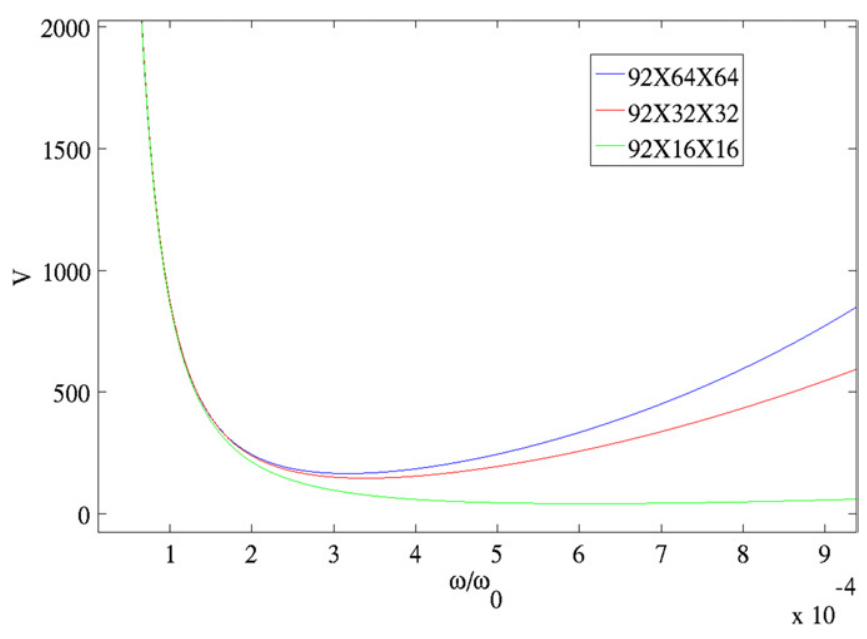

Figure 10. Value of the volume $V(\omega)$ for $\omega^{2} \ll\left|\omega_{\mathrm{MHD}_{\min }}^{2}\right|$ summing over $92 \times 64 \times 64$ intervals, $92 \times 32 \times 32$ intervals and $92 \times 16 \times 16$ intervals in the $\left(q, \alpha, \theta_{k}\right)$ space. Here $\omega_{0}=\sqrt{-\omega_{\mathrm{MHD}_{\min }}^{2}}$

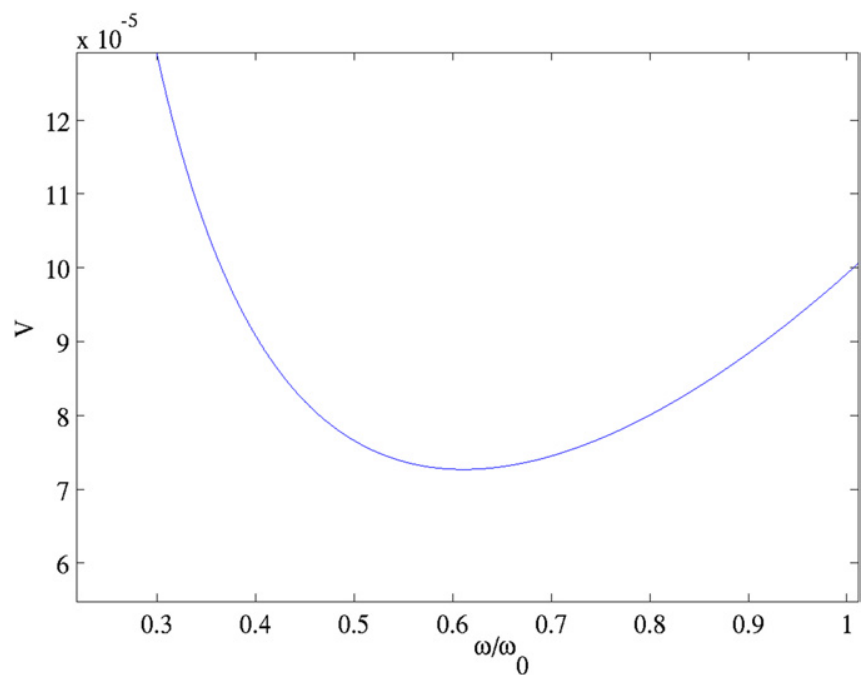

Figure 11. Value of the volume $V(\omega)$ for $\omega^{2} \rightarrow-\omega_{\mathrm{MHD}}^{2}$ and taking $\Omega_{\mathrm{h}}^{*}=0$. The integral was approximated by a sum over $92 \times 64 \times 64$ intervals in the $\left(q, \alpha, \theta_{k}\right)$ space. Here $\omega_{0}=\sqrt{-\omega_{\mathrm{MHD}_{\min }^{2}}^{2}}$.

a stellarator, mainly because stellarators tend to have much smaller toroidal currents than tokamaks. They can also be important in space and astrophysical plasma. We know from the MHD studies that a region of the plasma becomes unstable as soon as the local stability limit yields a negative $\omega_{\text {MHD }}^{2}$. But the MHD theory has some limits, and we know from previous research that the linear dynamics are not well represented in terms of the exponential growth of a single mode. That is why, in our work, we have considered a drift stabilized model. This gives a better insight into the physical reality. This work is a continuation of McMillan and Dewar's research [6]. We have used the same concepts they have employed, but extended the model by taking a drift stabilization term $\omega^{*}=\Omega_{\mathrm{pi}}^{*} k_{\alpha}+\Omega_{\mathrm{h}}^{*} / k_{\alpha}$ varying across the plasma volume and due to the presence of anisotropic hot ions. Furthermore, we considered the weakly interacting hot particle kinetic ballooning model. We 
considered a typical MHD equilibrium heated by tangential neutral beam injectors. This allowed us to compute more realistic growth rates and frequencies to model ballooning stability. Since the results we were looking for were discrete, we had to use adequate interpolation schemes to follow the ray trajectories in ballooning space. We obtained typical ray trajectories, investigated the dominating terms in the ray equations and computed the marginal stability using the Weyl formula. This study allows us to get a better insight into the drift stabilization effect, which is expected to have significant consequences for fusion experiments. Our initial data set was taken from experiments on the LHD stellarator which often operates in regimes predicted to be unstable in the ideal MHD limit. MHD activity is not generally observed in such a configuration and diamagnetic drift effects are expected to be sufficient to completely stabilize the device.

In the Weyl analysis, two branches were computed. The drift branch which has vanishing Weyl number at zero frequency and the more interesting MHD branch where the Weyl number remains finite. The minimum phase space volume for the MHD branch, hence the Weyl number, decreases to lower frequency when finite $\Omega_{\mathrm{h}}^{*}$ effects are taken into account, but is larger than that when $\Omega_{\mathrm{h}}^{*}=0$. Other kinetic effects should be examined in the future.

\section{References}

[1] Iiyoshi A et al 1999 Nucl. Fusion 391245

[2] Yamaguchi T et al 2005 Nucl. Fusion 45 L33

[3] Nakajima N 1996 Phys. Plasmas 34556

[4] Watanabe K Y et al 2005 Nucl. Fusion 451247

[5] Cooper W A 1982 Plasma Phys. 24265

[6] McMillan B F and Dewar R L 2006 Nucl. Fusion 46 477-86

[7] Yamagishi O, Nakamura Y and Kondo K 2003 Phys. Plasmas 102871

[8] Cooper W A 1983 Phys. Fluids 261830

[9] Dewar R L and Glasser A H 1983 Phys. Fluids 26 3038-52

[10] Catto P J, Tang W M and Baldwin D E 1981 Plasma Phys. 23639

[11] Brocher L, Cooper W A, Graves J P, Cooper G A, Narushima Y and Watanabe K Y 2010 Nucl. Fusion 50025009

[12] Cooper G A, Graves J P, Cooper W A, Gruber R and Peterson R S 2009 J. Comput. Phys. 228 4911-6

[13] Furth H P, Killeen J, Rosenbluth M N and Coppi B 1966 Plasma Physics and Controlled Nuclear Fusion Research (Vienna: IAEA) vol 1 p 103

[14] Greene J M and Johnson J L 1968 Plasma Phys. 10729

[15] Bernstein I B, Frieman E A, Kruskal M D and Kulsrud R M 1958 Proc. R. Soc. Lond. A 24417

[16] Cooper W A, Singleton D B and Dewar R L 1996 Phys. Plasma 3 275-80

[17] Gruber R, Cooper W A, Beniston M, Gengler M and Merazzi S 1991 Phys. Rep. 207 167-214 